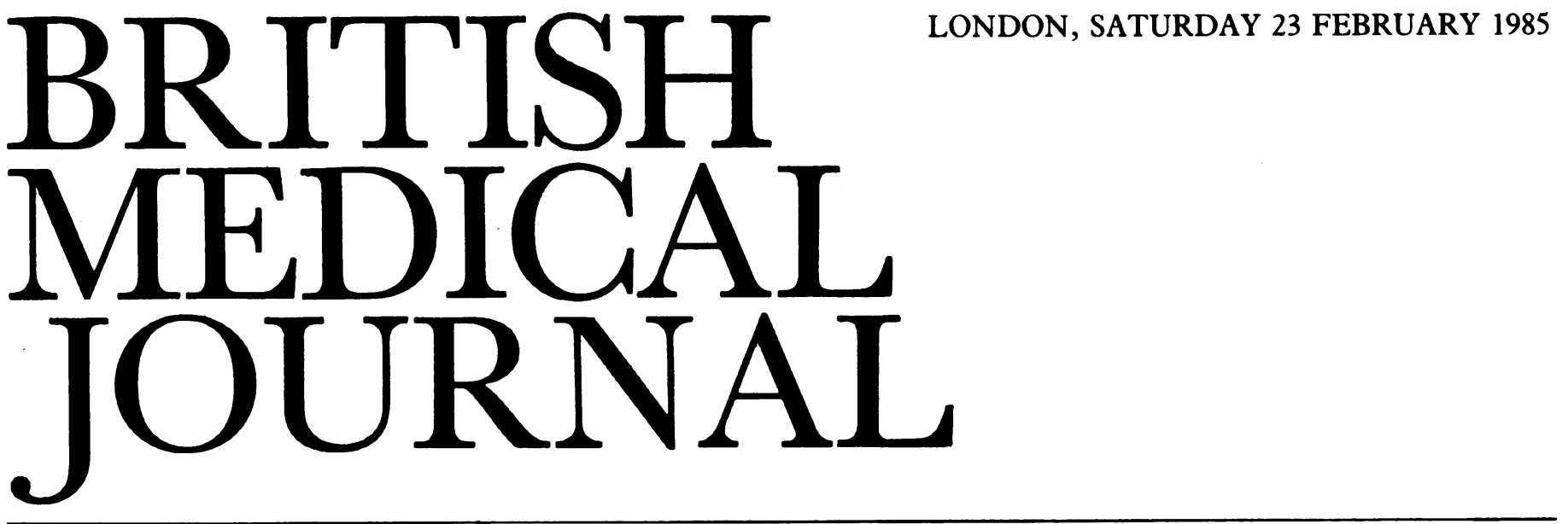

In its drive for economy the government has included among its targets the Public Health Laboratory Service of England and Wales. The proposal under consideration is the transfer of some (or possibly all) the Service's 52 re rional and area laboratories to the control of health authorities. The argument seems to be that in the existing joint PHLS-hospital laboratories most of the specimens examined are for the diagnosis of infection in individual patients, so that it would be logical to transfer these laboratories to the control of the National Health Service.

The joint PHLS-hospital laboratories became the pattern in the 1960s by agreement between the PHLS and the Department of Health and Social Security. The reasons were partly economic but mainly because joint laboratories alone could guarantee adequate ascertainment of the prevalent communicable diseases and so provide a basis for sound advice on effective measures for their prevention and control. Such advice can come only from properly coordinated and well planned epidemiological studies; and these in turn cannot begin without accurate information from sufficiently numerous and representative sources about what infections are prevalent.

The NHS has benefited from the links in many ways. The joint laboratories have been able to recruit well trained consultants and eager ambitious trainees learning their jobs and making up their minds about where their talents will best find fulfilment. The health authorities have generally received a better and cheaper service because of the central link with a professionally directed service, able and willing to answer problems quickly-often over the telephoneinstead of waiting for meetings of committees and subcommittees and references back. They have been able to do so because the relations are personal and professional and because of continuing awareness in the PHLS that, in control of infection, action delayed is action made futile.

Recently, however, cuts in the financial allocation to the PHLS have made it increasingly difficult to maintain a clinical microbiological service and a public health microbiological service in the 52 laboratories together with the support and coordination needed for national epidemiological work. Understandably, the clinical microbiological service to sick patients tends to be given precedence over the public health and epidemiological functions.

(C) BRITISH MEDICAL JOURNAL 1985. All reproduction rights reserved.
Furthermore, the creation of the Communicable Disease Surveillance Centre by the PHLS on behalf of the DHSS and the Welsh Office has required additional epidemiological resources for the national surveillance and control of communicable disease, functions previously performed by the departments. The laboratory reporting system has been expanded with contributions from hospital laboratories, individual consultants, and the Royal College of General Practitioners helping to provide a fuller epidemiological picture. Many more field investigations of outbreaks have been undertaken, as publications in the $B M \mathcal{F}$ have shown. The result has been an unfortunate decline in the multicentre studies and epidemiological research for which the PHLS structure is particularly suited.

So the PHLS is at a crossroads. Starved of funds, it can maintain its national laboratory service only at the expense of curtailing its epidemiological activities_or conversely maintain or increase its epidemiological activities only at the expense of the laboratory service. If the second course is chosen-as we believe it should be, given that the original purpose of the PHLS was to cope with the threat of epidemic infections in time of war-then the number of its laboratories might need to be cut. One way forward might then, however, be the creation of a network of regional epidemiologists based in laboratories throughout the country linked with a strong central unit.

What seems plain is that district health authorities have neither the incentive nor the powers to get going measures of action and investigation covering regions wider than their own. Which health authority will find money to send its chief medical microbiologists to four or five staff meetings a year for discussion of ideas for joint investigations and for joining working parties to get on with the job? It is from such staff meetings lasting a whole day that good propositions, often unexpectedly, emerge and effective teams are formed. The central reference laboratories concerned and the Communicable Disease Surveillance Centre work out details of methods and of recording and analysing results. It is only then that reliable answers become available in a short enough time to give good advice on prevention and control-advice often greatly welcomed and much needed by the Department of Health and Social Security. None of this beneficial work will continue if the link between the centre and the periphery 
is broken. Some laboratories may have to be transferred to health authority control, but the PHLS cannot function if too many of its roots are pruned.

The PHLS is a uniquely English institution, uniquely successful. It is admired and envied for its effective peripheral contacts by its counterpart in the United States, despite the vastly greater resources made available to the latter; and it is repeatedly consulted - and not in vain-by the World Health Organisation. Those who would break the essential PHLS links between centre and periphery-who are they? Do they really know what they are doing? The PHLS now has over 45 years of experience, accomplishment, and partnership with the DHSS and health authorities. Its essential structure must not be wrecked on the assumptioncertainly false - that real economies will result.

34 Redford Avenue,

JAMES HowIE

Edinburgh EH13 OBU

\section{The antiprogesterones are coming: menses induction, abortion, and labour?}

The five classes of steroid hormones are oestrogens, androgens, mineralocorticoids, progesterones, and glucocorticoids. As antagonists to these hormones have been developed they have proved of considerable clinical value. The actions of oestrogen may be inhibited by clomiphene or tamoxifen, androgens by cyproterone, and mineralocorticoids by spironolactone. Until now, however, no clinically acceptable receptor antagonists have been found for the progestogens or the glucocorticoids; but antiprogesterone drugs should soon be available and will give opportunities for the induction of menstruation and the interruption of pregnancy and may lead to a revision of our concepts of abortion.

Progesterone is a key hormone in the establishment and maintenance of human pregnancy. An antiprogesterone drug would therefore induce menstruation when administered in the luteal phase of the menstrual cycle, prevent implantation when administered at nidation, and induce abortion when administered in early pregnancy. Such compounds might revolutionise family planning, providing women with a non-surgical method of birth control when menstruation is delayed.

For some time steroid biochemists have been trying to produce potent competitive inhibitors of progesterone which are free of side effects. Earlier compounds (including gestrinone (R-2323), anordrin, and ORF 9371) were not clinically useful either because of androgenic side effects or simply because of their low efficacy when tested in vivo. ${ }^{1-3}$ The alternative approach of blocking the enzyme $3 \beta$ hydroxysteroid dehydrogenase by trilostane or epostane, thereby inhibiting the synthesis of progesterone and so inducing abortion, is a real advance-but unfortunately the same mechanism interferes with the biosynthesis of adrenal steroids. ${ }^{4}$

A fresh approach was presented by Philibert and colleagues in 1982.5 They reported the synthesis of a new steroid compound, RU 486, which is a derivative of the progestogen norethindrone. In female rabbits pretreated with oestradiol this antiprogesterone bound to progesterone receptors five times more avidly than did progesterone, and also bound three times more strongly than dexamethasone to the glucocorticoid receptor of the thymus in adrenalectomised rats. Despite such binding this synthetic steroid had no progestomimetic activity in bioassay systems in vitro or in vivo. This suggested that RU 486 would be clinically useful as an antiprogesterone; and studies in monkeys showed that RU 486 reliably induced menstruation by a local action on the endometrium. ${ }^{6}$ This action was seen with doses as low as $0.1 \mathrm{mg} / \mathrm{kg}$ given intramuscularly. The corpus luteum was not concerned in this response, and RU 486 did not bind to the primate ovary.

Three studies have reported the use of RU 486 to abort human pregnancies. Herrmann and associates gave $200 \mathrm{mg}$ of RU 486 a day by mouth for four days to women who were six to eight weeks pregnant and produced abortion in nine of 11 patients. ${ }^{7}$ The median time to abortion was four days (range three to seven days). One of the patients experienced heavy bleeding and required emergency curettage. More recently, in a collaborative study by the World Health Organisation, this antiprogesterone was given to 37 women seeking termination of pregnancy with amenorrhoea of 42 days or less. ${ }^{8}$ The patients were given either $25 \mathrm{mg}$ RU 486 twice daily for four days (total dose $200 \mathrm{mg}$ ), $50 \mathrm{mg}$ twice daily for four days (400 $\mathrm{mg}$ ), or $100 \mathrm{mg}$ twice a day for four days $(800 \mathrm{mg})$. Twenty two of the women had a complete abortion while 11 had an incomplete abortion, and in the remaining three there was apparent continuation of the pregnancy. At these doses the clinical efficacy of treatment was not dose dependent. Plasma progesterone concentrations did not decline greatly during treatment with antiprogesterone, though plasma cortisol concentrations did increase. Two patients in this study had heavy bleeding and required a blood transfusion and curettage on clinical grounds, but no patient complained of severe pain, nausea, or vomiting after the antiprogesterone-in contrast with early studies of various prostaglandin regimens. Despite these advantages these early results do not show any clinical advantage over vacuum aspiration or prostaglandin analogue methods for terminating early pregnancy.

One way to increase the percentage of abortions that are complete would be to use an antiprogesterone to dislodge the pregnancy and then to give a prostaglandin to evacuate the uterus. Bygdeman and colleagues recently prescribed RU 486 at $25 \mathrm{mg}$ twice daily for four days (200 mg), or 25 mg four times daily for four days ( $400 \mathrm{mg}$ ), followed by a single intramuscular injection of $250 \mu \mathrm{g}$ sulprostone (15methylprostaglandin $E_{2 a}$ methyl ester) to 16 pregnant patients with amenorrhoea of 49 days or less. ${ }^{9}$ With this combined regimen all women had complete abortions. No patient reported serious vomiting or diarrhoea, and only one woman required an analgesic injection. Such combined treatment, aiming at giving low doses of two drugs working by different mechanisms to produce the same clinical effect, has the same rationale as using diuretics and $\beta$ blockers in treating hypertension.

Antiprogesterone steroids might also act as antifertility agents in other circumstances. Ingestion from day 24 to day 26 of a normal ovarian/menstrual cycle, regardless of whether conception has occurred, will induce menstruation at the expected time - and in contrast with conventional oral contraceptives would limit the duration of drug treatment to only two or three days in each menstrual cycle. Giving an antiprogesterone to a patient whose expected menses are overdue but in whom a routine pregnancy test gives a 